\title{
Space-Time Development of Electromagnetic and Hadronic Showers and Perspectives for Novel Calorimetric Techniques
}

\author{
Andrea Benaglia, Etiennette Auffray, Member, IEEE, Paul Lecoq, Fellow, IEEE, Hans Wenzel, and Adam Para
}

\begin{abstract}
The performance of hadronic calorimeters will be a key parameter at the next generation of High Energy Physics accelerators. A detector combining fine granularity with excellent timing information would prove beneficial for the reconstruction of both jets and electromagnetic particles with high energy resolution. In this work, the space and time structure of high energy showers is studied by means of a GEANT4-based simulation toolkit. In particular, the relevant time scales of the different physics phenomena contributing to the energy loss are investigated. A correlation between the fluctuations of the energy deposition of high energy hadrons and the time development of the showers is observed, which allows for an event-by-event correction to be computed to improve the energy resolution of the calorimeter. These studies are intended to set the basic requirements for the development of a new-concept, total absorption time-imaging calorimeter, which seems now within reach thanks to major technological advancements in the production of fast scintillating materials and compact photodetectors.
\end{abstract}

Index Terms-hadron calorimetry, timing, dual readout, simulation.

\section{INTRODUCTION}

A MONG the biggest challenges for future High Energy Physics experiments, the capability of measuring hadronic jet energies with high precision will be a crucial one. An oft-quoted physics case driving the level of precision needed is the separation of $\mathrm{W}$ and $\mathrm{Z}$ bosons via their invariant mass reconstruction in di-jet final states. To achieve this, a single jet energy resolution better than $4 \%$ is required, which largely exceeds the performance of today's detectors. Following the Higgs boson discovery in 2012, good jet energy resolution will also be crucial to tag Higgs bosons produced in association with top quarks or vector bosons decaying hadronically. Multi-jet final states are also expected by many different Beyond Standard Model theories in which new heavy particles are foreseen to decay with the emission of $\mathrm{W}$ and $\mathrm{Z}$ bosons, and their reconstruction in the hadronic decay modes will play a major role. Novel approaches to hadronic calorimetry are currently being investigated, the particle flow approach (PFA) and the dual readout approach (DR) being

The research leading to these results has received funding from the European Research Council under the European Union's Seventh Framework Programme (FP/2007-2013) under ERC Grant Agreement n. 338953-TICAL and under Grant Agreement 289355-PicoSEC-MCNet. Fermilab is operated by Fermi Research Alliance, LLC under Contract No. DE-AC02-07CH11359 with the United States Department of Energy.

A. Benaglia (corresponding author), E. Auffray, and P. Lecoq are with CERN, Genève, Switzerland (e-mail: andrea.benaglia@ cern.ch).

A. Para and H. Wenzel are with Fermi National Accelerator Laboratories, Batavia (IL), USA (e-mail: para@fnal.gov). the two main complementary techniques contemplated. The former [1] aims at reconstructing each single particle in a jet, thus profiting from the excellent energy resolution of tracker and electromagnetic calorimeter for the energy measurement of charged particles and photons within the jet respectively. The latter [2] strives to improve the hadronic energy resolution by correcting for fluctuations of the shower composition on an event-by-event basis, thanks to the simultaneous measurement of the total energy deposition through scintillation light and the electromagnetic component through Cherenkov light. Significant advances in the production of dense inorganic scintillators [3] and compact photodetectors [4] open the way to the development of a third approach, a new-concept, total absorption crystal calorimeter combining fine granularity and excellent time resolution with dual readout capabilities at the level of each individual calorimeter cell. In this report, an investigation of the potential use of the time dimension in hadron energy reconstruction is presented. After a description of the simulation techniques in section II and different time definitions in section III, the time structure of electromagnetic and hadronic showers is discussed in section IV. Finally, section $\mathrm{V}$ describes a possible technique to use the timing information to correct for fluctuations in the energy deposition process, alternative to standard DR techniques.

\section{The Simulation FramewORK}

A dedicated simulation toolkit, CaTs, was developed to allow for a detailed study of the time and space properties of high energy electromagnetic and hadronic showers in a calorimeter. CaTs [5], [6] (Calorimeter and Tracker Simulation) is a flexible, standalone application based on GEANT4 [7] and RoOT [8]. In CaTs, detector geometries are implemented using the XML based Geometry Description Markup Language (GDML) [9]. The detector segmentation, the material properties and composition, as well as the material optical properties can be customized according to the user's needs. Specific Sensitive Detector classes, together with the corresponding Hit Collections, are implemented to define the detector response to the passage of particles. For the purposes of this study, a sensitive detector class representing a DR calorimeter was used. In each calorimeter cell, the sum of the energy deposits of all particles traversing that sensitive volume is registered, as well as the number of Cherenkov photons produced by charged particles above Cherenkov threshold. A more detailed breakdown of energy deposits and Cherenkov 
photons per particle type and underlying physics process is also written out to the event stream.

\section{A. Adding time information}

To allow timing studies, the basic functionality of CaTs was extended in order to include the time dimension. Time bins of adjustable size were introduced, so that a detector hit could be defined as the sum of all energy deposits in a specific calorimeter cell occurring in a specific time interval. Given the different time scales of the physics processes responsible for the development of a hadron shower (ranging from less than a ns up to a few $\mu$ s), it proved effective to define three different time ranges:

- time bins of $10 \mathrm{ps}$ size between 0 and $5 \mathrm{~ns}$;

- time bins of $200 \mathrm{ps}$ size between 0 and $100 \mathrm{~ns}$;

- time bins of $10 \mathrm{~ns}$ size between 0 and $5 \mu$ s.

\section{B. Further details about the simulation}

Version 4.10.1.patch-01 of GEANT4 released on March $27^{\text {th }}, 2015$ was used for all the studies in this report. The QGSP_BERT_HP was used as reference physics list, with HP indicating the data-driven high precision neutron package used to transport neutrons below $20 \mathrm{MeV}$ down to thermal energies. A discussion on the measurement of timing in hadronic showers in and the validation of different GEANT4 hadronic models can be found in [10].

\section{DifFERENT TIME DEFINITIONS}

The time associated with a signal recorded in a cell of a calorimetric detector can be defined in multiple ways. In a first natural approach, the frame of reference being the detector as a hole, the time of a hit is simply defined as the time passed between a reference time $t_{0}$ (e.g. the instant the primary particle enters the detector volume) and the energy deposition in the detector. In the following, it will be indicated as $t_{\text {global }}$. A second possible approach involves a cell-based time definition, in which the global time associated with a hit is corrected for the time of flight of a particle moving at the speed of light $c$ to get to the depth of that specific cell in the detector along the beam axis. If $z_{\text {cell }}$ is the cell distance from the detector front face along the beam axis, the local time is defined as $t_{\text {local }}=t_{\text {global }}-z_{\text {cell }} / c$. The difference between the global and local time definition is mostly relevant at short time scales (i.e. < few nanoseconds) and for hadronic showers, that can extend over long distances in a calorimeter (up to few meters). Considering the core of a hadron shower that propagates at a speed close to that of light, the sub-ns component in $t_{\text {local }}$ gets diluted over several nanoseconds in $t_{\text {global }}$ due to the shower longitudinal extent, as shown in Fig. 1.

\section{Time Profiles of Electromagnetic And HADRONIC SHOWERS}

With the tools described in section II and the time definitions of III, the time structure of electromagnetic and hadronic showers was inspected. Space-time development of hadronic
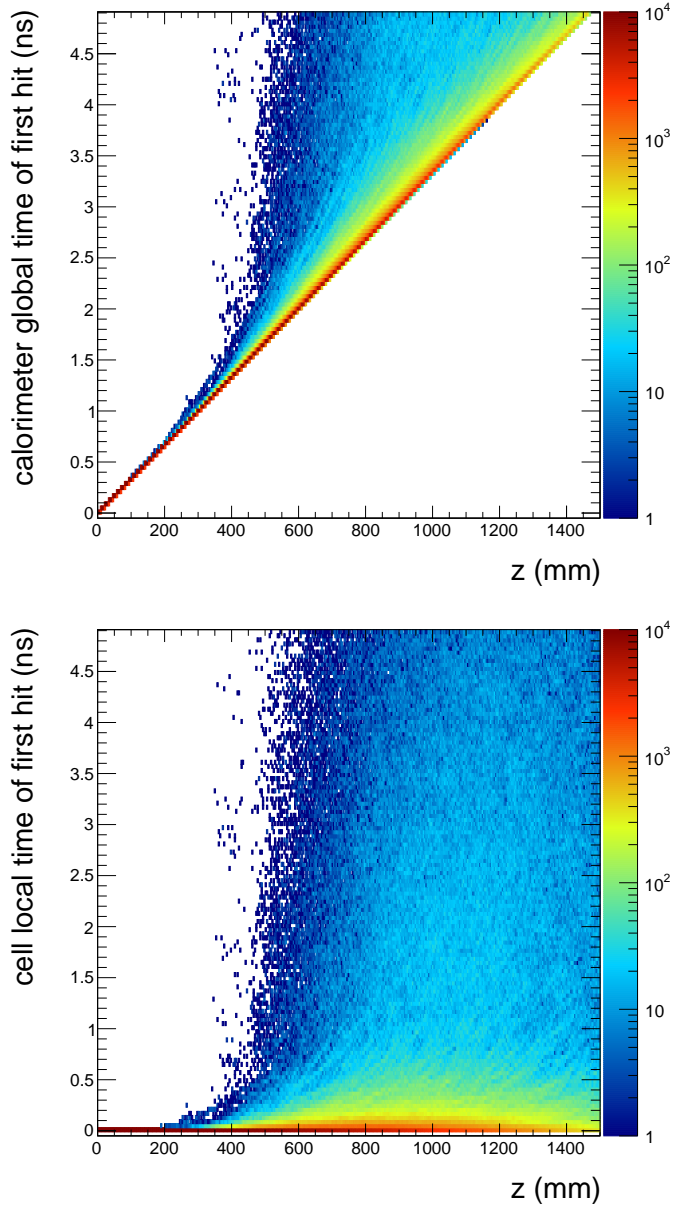

Fig. 1. Scatter plots of the time associated to the earliest hit of a detector cell vs. the distance of the cell from the detector front face along the beam $z$-axis for all the cells in the detector, for a $50 \mathrm{GeV}$ proton beam. The detector is made of a fully sensitive $1 \mathrm{~m} \times 1 \mathrm{~m} \times 3 \mathrm{~m}$ iron block, constituted by $1 \mathrm{~m} \times 1 \mathrm{~m} \times 1 \mathrm{~mm}$ adjacent cells. A global (local) time definition is assumed in the top (bottom) figure respectively.

showers and the composition of the underlying physics processes depends very weakly on the medium of the calorimeter (except for the evolution of the neutron-related component of hadronic showers). In this paper we use iron as a representative detector material, with the resulting performance of a detector constructed from any other material, like BGO, being nearly identical. In particular, a fully sensitive iron block divided into $30001 \mathrm{~m} \times 1 \mathrm{~m} \times 1 \mathrm{~mm}$ adjacent cells along the $z$-axis was used. Fig. 2 shows the time evolution of $50 \mathrm{GeV}$ electron, pion and proton showers, i.e. the amount of energy deposited by the shower throughout the whole detector for each time bin (the smallest time bin size of $10 \mathrm{ps}$ is considered). From the bottom plot, it is evident that both electromagnetic and hadronic showers are characterized by a very fast component, with a large fraction of energy deposited within the first tens of picoseconds of each cell's $t_{\text {local }}$. This means that most of the energy is carried by particles moving at a speed close to that of light, and this is true for hadrons too. The shower extent in the detector $-\sim 20$ to $30 \mathrm{~cm}$ for electrons, 1 to $1.5 \mathrm{~m}$ for pions and protons - extends the time profile to about $1 \mathrm{~ns}$, 


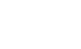
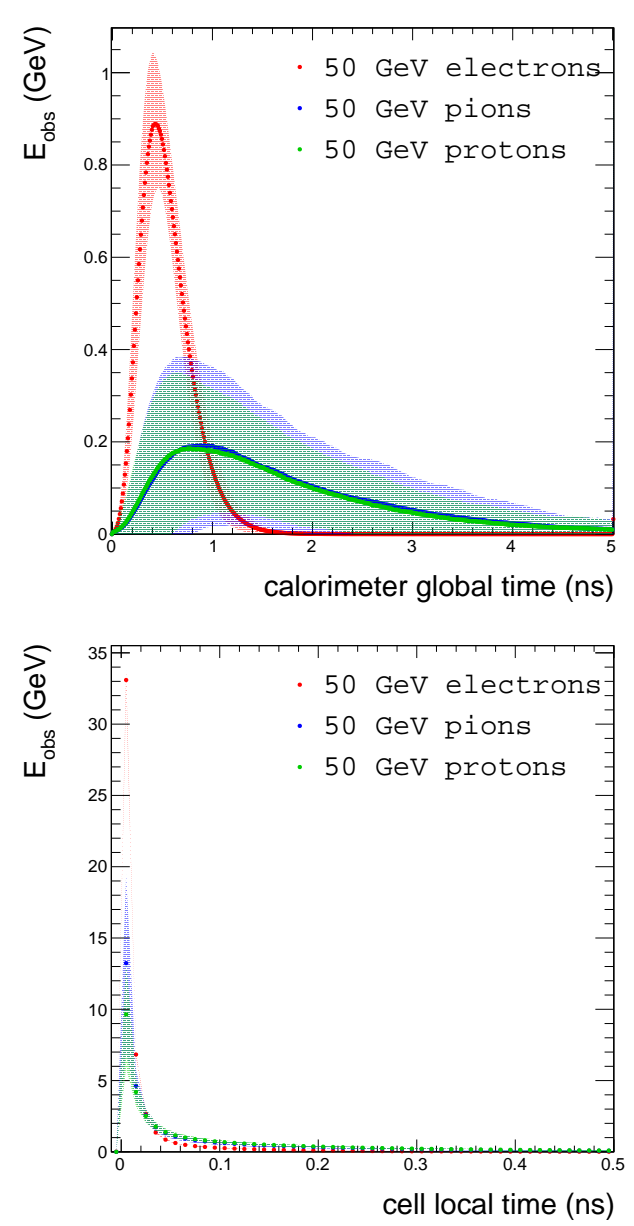

Fig. 2. Time evolution of electromagnetic (electrons) and hadronic showers (pions and protons). In the top figure, a detector global time definition is assumed, whereas in the bottom one the cell local time as defined in section III is used. In these and in the following figures, solid dots and shaded bands represent the mean value and the statistical uncertainty respectively.

for electrons and $4 \mathrm{~ns}$ for hadrons when a $t_{\text {global }}$ definition is considered, as can be seen in the top figure. For a more quantitative analysis, the cumulative distributions are shown in Fig. 3. The full evolution of an electromagnetic shower lasts for approximately $500 \mathrm{ps}$ in $t_{\text {local }}$, whereas a pion or a proton deposits about the $80 \%$ of its total energy release in the detector in the same amount of time. Half of the energy of an hadron shower is released in less than only $100 \mathrm{ps.} \mathrm{Notice}$ that in the analysis, a maximum time window of $10 \mu \mathrm{s}$ is considered. Therefore, by total energy deposit (labelled as $E_{\text {obs }}$ in the figures) we mean the energy deposited within the first $10 \mu$ s since the beam particle enters in the calorimeter. The actual energy deposit beyond this time is anyway negligible.

\section{ENERGY - TIME CORRELATION}

As seen in the previous section, a significant fraction of the energy of a hadron shower is promptly released in a calorimeter, although this fraction is subject to large event-byevent fluctuations due to the different relative contribution of the various physics processes responsible for the interaction of
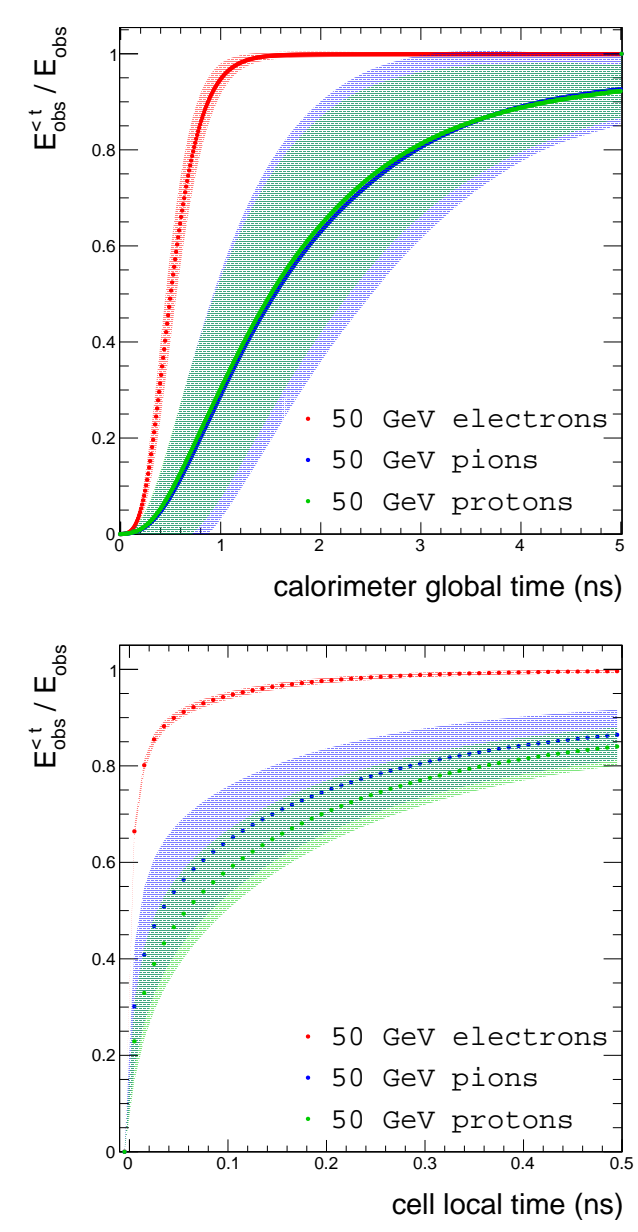

Fig. 3. Cumulative distribution of the time evolution of electromagnetic (electrons) and hadronic showers (pions and protons) shown in 2. The $y$ axis $\left(E_{\mathrm{obs}}^{<t} / E_{\mathrm{obs}}\right)$ represents the fraction of the shower energy deposited within a time $t$ over the total energy deposited in the calorimeter, assuming the moment when the beam particle enters the detector as $t_{0}$. In the top figure, a detector global time definition is assumed, whereas in the bottom one $t_{\text {local }}$ used.

hadrons in matter and their typical time scale. Three different time ranges can be roughly identified.

- $\lesssim 1$ ns: electromagnetic decays of $\pi^{0}$ 's and $\eta^{0}$ 's into photon pairs, as well as the direct ionization of high energy charged hadrons (such as $\mathrm{p}^{ \pm}$'s, $\pi^{ \pm}$'s, $\mathrm{K}^{ \pm}$'s) contribute a prompt, complete energy transfer to the calorimetric medium. On the other hand, hadron-nucleus interactions, whose main products are nucleons and nuclear fragments, cause a variable amount of energy to remain undetected. As a matter of fact, every nucleon extracted from the nucleus consumes about $8 \mathrm{MeV}$ of the shower energy to overcome its binding energy: given the large - but fluctuating - number of nucleons produced in each hadron shower, this amount of invisible energy turns out to be the most important source of degradation for the energy resolution. Electron/positron pairs from gamma conversions following nuclear de-excitation are also present.

- $\lesssim 100 \mathrm{~ns}$ : in this time range, delayed energy depositions are dominated by the ionization of the decay products of unstable particles, such as muons from kaon and pion 
decays and electrons or positrons from muon decays; in addition to that, fast neutrons produced in prompt nuclear interactions can either thermalize by scattering off nuclei or induce secondary nuclear interactions.

- $\gtrsim 100$ ns: nuclear capture of thermalized neutrons can take up to several $\mu$ s. The neutron energy is finally released in the detector by electrons and positrons from gamma conversions following neutron capture.

The observation above highlights that nuclear interactions are at the same time the dominant source of late energy depositions as well as the main cause for hadron poor energy resolution. Such a correlation between the fluctuations in the energy deposition of a hadron shower and its time evolution is illustrated in Fig. 4: $50 \mathrm{GeV}$ proton events were divided into different classes, depending on the shower total energy deposit, and the shower time profile was studied independently for each of them. Events with a smaller amount of energy released in the calorimeter were observed to be correlated with a slower evolution of the shower, and vice versa. This suggests that timing can be used to correct for energy fluctuations in a DRlike approach, as explained in the next subsection.

\section{A. A time-based correction}

A simple variable expressing the "speed" of the evolution of the shower is the fraction of the shower energy deposited within a cell local time $T$ over the total energy deposited in the calorimeter, in formulae $E_{\mathrm{obs}}^{t<T} / E_{\mathrm{obs}}:=x$. In order to obtain a correction function for the energy fluctuations, the ratio of the total deposited $\left(E_{\text {obs }}\right)$ to the true energy $\left(E_{\text {beam }}\right)$ was plotted as a function of the above variable, as is shown in Fig. 5 (bottom) for a simulated $50 \mathrm{GeV}$ pion beam, and assuming $T=1.25 \mathrm{~ns}$ (the choice of this value for $T$ will be justified in section V-C. For comparison to a standard DR approach [11], Fig. 5 (top) shows the $E_{\text {obs }} / E_{\text {beam }}$ ratio as a function of the Cherenkov-to-scintillation signal ratio (normalized in such a way that $C / S=1$ for an electron beam). In both cases, pion showers with a high electromagnetic fraction exhibit an electron-like behavior, with almost all the energy promptly deposited in the detector and a $C / S$ ratio approaching unity.

The observed correlation was parametrized, and the resulting fit function $f$ used to compute a multiplicative correction factor $1 / f(x)$ to be applied to the total energy deposit, where $x$ is computed on an event-by-event basis. The result is shown in Fig. 6, where the energy resolution of a pion beam is reported, as predicted by the simulation, for different cases: black solid points show the performance of a total absorption calorimeter where no further correction is applied in the energy reconstruction; red solid points refer to the same detector, where energy is corrected for using the time-based approach discussed above; the performance after a conventional DR correction is represented by blue squares. The measured performance of the CMS hadronic calorimeter at a pion beam test is also reported for reference (dashed line) [12]. The effect of a totally active detector with respect to a standard sampling calorimeter (e.g. CMS HCAL) is mostly visible in the stochastic term of the energy resolution, that can be as low as $\sim 20 \%$ for the former, while it is in the $60-100 \%$
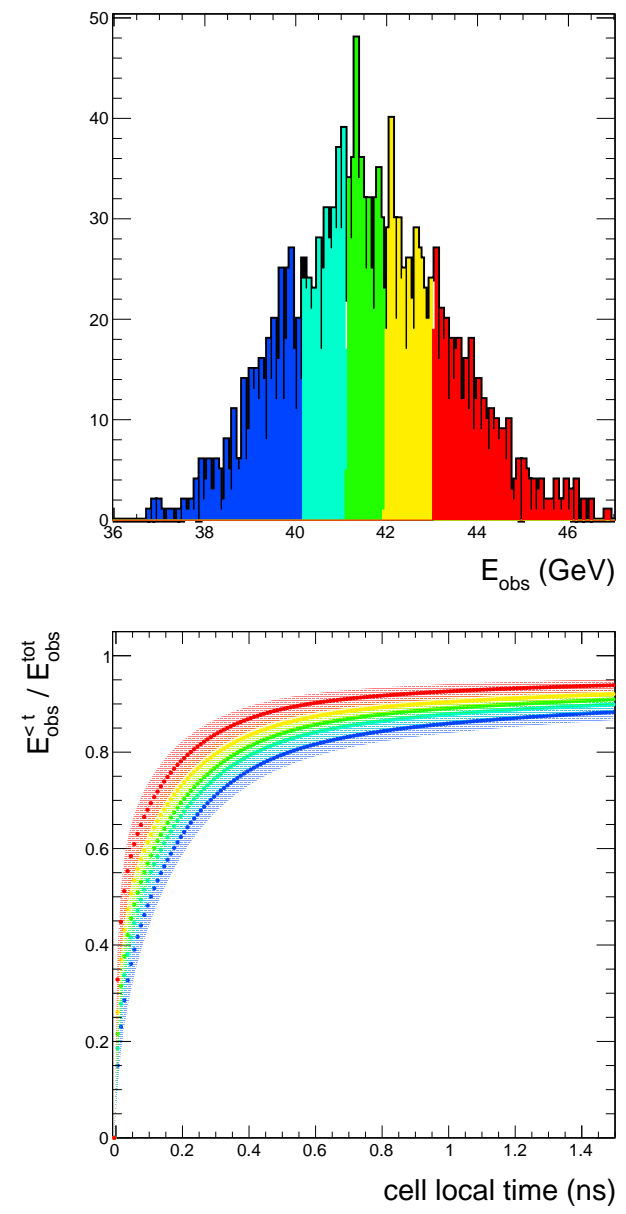

Fig. 4. Top figure: histogram showing the total energy deposit of $50 \mathrm{GeV}$ protons in a calorimeter (a fully sensitive $1 \mathrm{~m} \times 1 \mathrm{~m} \times 3 \mathrm{~m}$ iron block). The different colors identify five different event classes (from events marked in blue, depositing a smaller-than-average amount of energy, to events marked in red, which deposit a considerably larger fraction of energy in the detector). Bottom figure: for each of the previously defined event classes, the time evolution of the shower is represented, assuming a $t_{\text {local }}$ definition.

range for the latter. When a detector is able to provide further information, allowing for an event-by-event correction for the invisible energy, not only the stochastic term can further improve to $\sim 15 \%$, but more importantly the constant term of the energy resolution vanishes, thus allowing for very precise measurement at high energies. Simulation results indicate that timing can be a powerful variable in the reconstruction of hadron energies, offering an interesting alternative to Cherenkov-based DR approaches, and with comparable performances. These results were obtained with the reference detector described in section IV, i.e. with a longitudinal cell segmentation of $1 \mathrm{~mm}$. Further tests were made where the cell longitudinal size was increased to $3 \mathrm{~cm}$, but no appreciable worsening of the performance of the correction was observed.

\section{B. Detector response linearity}

High energy jets consist of a collection of particles of different species with a wide range of energies. Non-linearity of the calorimeter response and/or differences in the response to different particles, especially charged and neutral pions, induce 

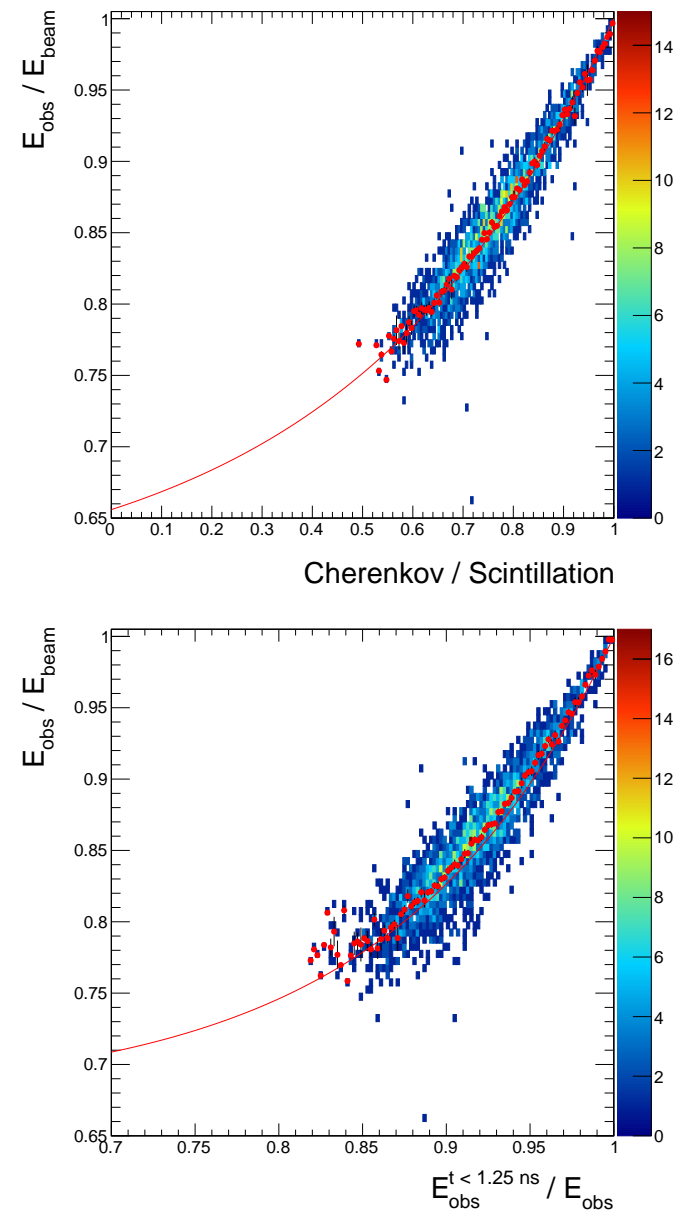

Fig. 5. Top figure: scatter plot representing the ratio of the total deposited $\left(E_{\text {obs }}\right)$ to the true energy $\left(E_{\text {beam }}\right)$ as a function of the Cherenkov-toscintillation ratio for $50 \mathrm{GeV}$ pions, as predicted by the simulation. Bottom figure: same scatter plot as above, where the variable on the $x$-axis is replaced by the ratio of the energy deposited in the calorimeter within the first $1.25 \mathrm{~ns}$ (in $t_{\text {local }}$ ) to the total energy deposited in the calorimeter.

a major degradation of the energy resolution for hadronic jets. The time-based correction described in the previous section corrects the response of the calorimeter to hadrons to a level very close to the response to electrons, as shown in Fig. 7. With the same, energy independent correction function applied to pion and proton showers, the response of the detector in the range 1 to $100 \mathrm{GeV}$ is within $\sim 2.5 \%$ and the responses to charged pions or protons are equal within $\pm 2.5 \%$.

\section{Optimization of the integration time window}

The results above discussed depend on the value of the integration time window $T$ over which energy deposits in the calorimeter are added up to get the detector prompt response. The estimator that was used to get the optimal working point is the energy resolution extracted from the simulation after a time-based energy correction computed with different values of $T$ was applied. The result, shown in Fig. 8, indicates that the best performance in terms of energy reconstruction is attained when a time integration window of about $1 \mathrm{~ns}$ is used, with a significant improvement in the stochastic and especially in the constant term of the energy resolution.

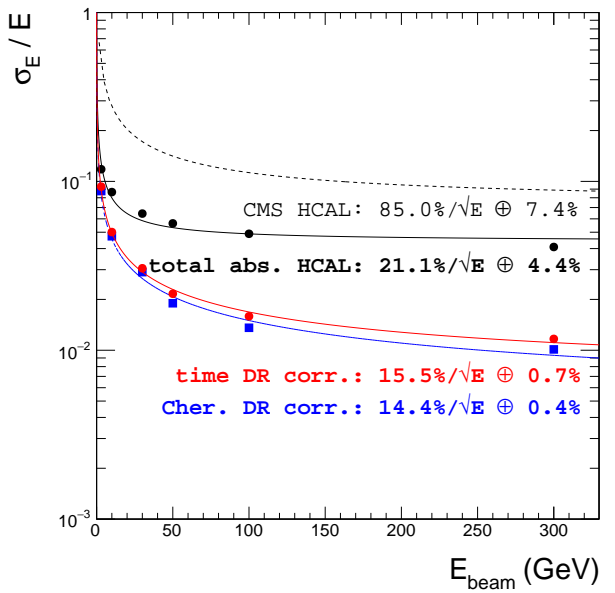

Fig. 6. Energy resolution for pion beams. Black solid dots represent a fully sensitive detector $(1 \mathrm{~m} \times 1 \mathrm{~m} \times 3 \mathrm{~m}$ iron block); red solid dots represent the same detector when a time-based energy correction is applied; blue solid squares refer to a Cherenkov-to-scintillation DR correction. The response of the CMS HCAL to pions as measured at a beam test is represented by the dashed line.

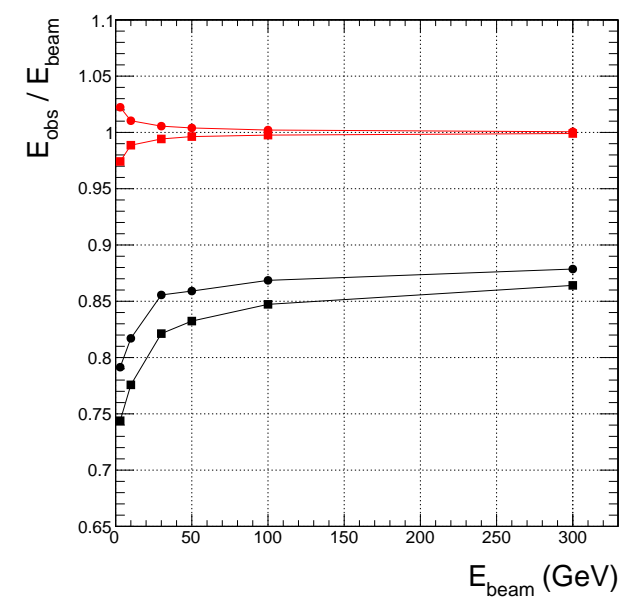

Fig. 7. Linearity of the energy response of the simulated calorimeter. Solid black points and squares represent the average of the energy deposited in the detector by pions and protons respectively divided by the beam energy when no further corrections are applied. Solid red dots and squares refer to the case where the deposited energy is corrected for using the timing information as described in section V-A. The same correction function is used for both pions and protons.

\section{CONCLUSION}

The initial studies of the space-time development of hadronic and electromagnetic showers with the modified CaTs framework indicate that there a significant improvement in the performance of the hadron calorimeters can be achieved using the timing information from a detector. The fraction of the shower energy deposited by a fast component can be used in a similar manner to that proposed by DR calorimetry to correct for the fluctuations of energy loss due to nuclear effects, and leading to greatly improved energy resolution and response linearity of hadron calorimeters. The use of the timing information may present much easier technical challenges that the detection of the Cherenkov component of 


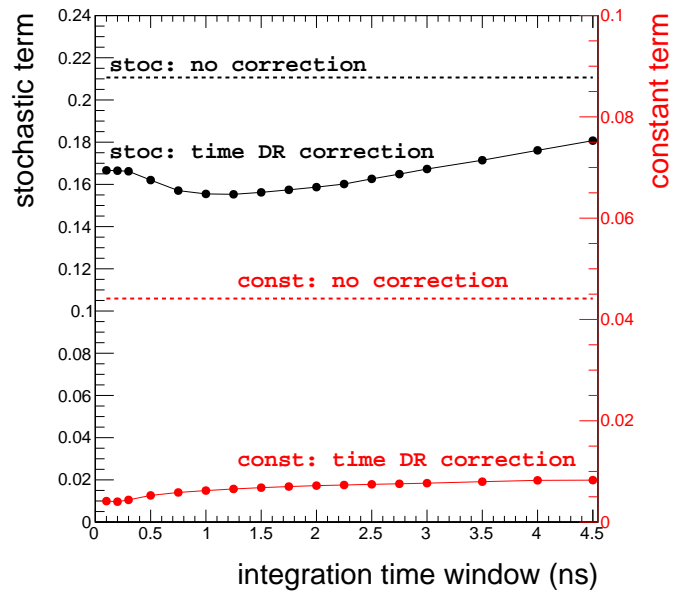

Fig. 8. Optimization study of the integration time window $T$ of the variable $x=E_{\mathrm{obs}}^{t<T} / E_{\mathrm{obs}}^{t<10 \mu \mathrm{s}}$, used in the computation of the time-based energy correction. The stochastic and constant terms of the energy resolution attained with each of the different values of $T$ tested are shown in black and red respectively. The performance of the simulated detector when no energy correction is applied is shown as well (dashed lines).

the shower.

\section{REFERENCES}

[1] M.A. Thomson, "Particle Flow Calorimetry and the PandoraPFA Algorithm," Nucl. Instrum. Meth., vol. A611, pp. 25-40, 2009.

[2] N. Akchurin et al., "Dual-readout Calorimetry," Nucl. Instrum. Meth., vol. A550, pp. 185-200, 2005.

[3] P. Lecoq, "Development of new scintillators for medical applications," Nucl. Instrum. Meth., vol. A, 2015.

[4] "New Developments in Photodetection NDIP14," Nucl. Instrum. Meth., vol. A787, pp. 1-398, 2015, NDIP14 conference proceedings.

[5] H. Wenzel, "Simulation studies of a total absorption dual readout calorimeter," J. Phys. Conf. Ser., vol. 404 012049, pp. 7, 2012.

[6] http://home.fnal.gov/wenzel/CaTS.html.

[7] S. Agostinelli et al., "GEANT4: a simulation toolkit," NIM A, vol. 506, no. 3, pp. 250-303, 2003 and J. Allison et al., "GEANT4 Developments and Applications," IEEE Trans. Nucl. Sci., vol. 53, no. 1, pp. 270-278, 2006.

[8] R. Brun and F. Rademakers, "Root - An object oriented data analysis framework," NIM A, vol. 389, no. 1-2, pp. 81-86, 1997.

[9] http://lcgapp.cern.ch/project/simu/framework/GDML/gdml.html.

[10] C. Adloff et al., "The time structure of hadronic showers in highly granular calorimeters with tungsten and steel absorbers," JINST, 9, P07022, 2014.

[11] K. Genser, A. Para, and H. Wenzel, "Very High Resolution Hadron Calorimetry," IEEE Trans. Nucl. Sci., vol. 53, no. 1, pp. 1177-1182, 2011.

[12] The CMS Collaboration, "The CMS hadron calorimeter project: Technical Design Report," CERN-LHCC-97-031, pp.451, 1997. 